Bull. Austral. Math. Soc.

65R20, 45G10

VoL. 38 (1988) [151-152]

\title{
A NEW COLLOCATION-TYPE METHOD FOR THE NUMERICAL SOLUTION OF HAMMERSTEIN EQUATIONS SUNIL KUMAR
}

The new method approximates the solution of the Hammerstein equation

$$
y(t)=f(t)+\int_{a}^{b} k(t, s) g(s, y(s)) d s, \quad t \in[a, b]
$$

by first applying the standard collocation method to an equivalent equation for $z(t):=$ $g(t, y(t))$. It then obtains the approximation to $y$ by using the equation

$$
y(t)=f(t)+\int_{a}^{b} k(t, s) z(s) d s, \quad t \in[a, b] .
$$

Let $z_{n}$ and $y_{n}$ be the approximations to $z$ and $y$ respectively. It is proved that, under suitable conditions, $y_{n}$ converges to $y$ at a rate at least equal to that of the best approximation to $z$ from the space in which $z_{n}$ is sought. In particular, it is shown that if $z_{n}$ is sought in certain piecewise polynomial function spaces, then $y_{n}$ may exhibit (global) superconvergence, that is, it may converge to $y$ at a faster rate than $z_{n}$ does to $z$.

The discrete version of the method, which arises when numerical quadrature is used to approximate required integrals, is analysed for interpolatory quadrature rules and piecewise polynomial approximations to $z$. The analysis shows that the discrete approximation to $z$ has the same order of convergence as its 'exact' counterpart if $k$ is sufficiently smooth, and if the quadrature rule used is of sufficient precision.

The new method is used in the computation of a simple turning point $\left(y=y^{c}, \lambda=\lambda^{c}\right)$ of the parameter-dependent equation

$$
y(t)=f(t)+\lambda \int_{a}^{b} k(t, s) g(s, y(s)) d s, \quad t \in[a, b]
$$

where $\lambda \in \mathbf{R}$. In the spirit of the method, the simple turning point $\left(z=z^{c}, \lambda=\lambda^{c}\right)$ of an equivalent equation for $z(t):=\lambda g(t, y(t))$ is computed first. This involves the

Received 9 December 1987. Thesis submitted to the University of New South Wales, April 1987. Degree approved November, 1987. Supervisor: Professor I.H. Sloan.

Copyright Clearance Centre, Inc. Serial-fee code: 0004-9729/88 \$A2.00+0.00. 
application of the standard piecewise polynomial collocation method to a certain system of equations which has $\left(z^{c}, \lambda^{c}\right)$ as part of an isolated solution.

Let $\left(z_{n}, \lambda_{n}\right)$ denote the approximation to $\left(z^{c}, \lambda^{c}\right)$. Then $y_{n}$, the approximation to $y^{c}$, is obtained as before by the use of (2). It is proved that, under suitable conditions, the approximations to $y^{c}$ and $\lambda^{c}$ are both superconvergent, that is, they both converge to their respective exact values at a faster rate than $z_{n}$ does to $z^{\mathrm{c}}$.

Finally, (1) is considered again, and uniform convergence of $y_{n}$ to $y$ is established for the case where $z_{n}$ is a polynomial of degree $\leqslant n-1$, with coefficients determined via collocation at the zeros of the $n$th degree Chebyshev polynomial of the first kind.

Computer Centre

The University of the South Pacific

P.O. Box 1168

Suva

Fiji 